\title{
The use of alternative polyadenylation sites renders $\beta 1$ - integrin mRNA isoforms with differential stability during mammary gland development
}

\author{
Julian Naipauer ${ }^{1,3 *}$, Albana Gattelli ${ }^{2,3 *}$, Maria Sol Degese ${ }^{1,3}$, \\ Victoria Slomiansky ${ }^{2,3}$, Eva Wertheimer ${ }^{4}$, Jonathan LaMarre ${ }^{5}$, Lucio Castilla ${ }^{6}$, \\ Martin Abba ${ }^{7}$, Edith Claudia Kordon ${ }^{2,3} \dagger \ddagger \&$ Omar Adrian Coso ${ }^{1,3} \ddagger$.
}

${ }^{1}$ LFBM-DFBMC, Departamento de Fisiología y Biología Molecular, Facultad de Ciencias Exactas y Naturales, Universidad de Buenos Aires (FCEN - UBA),

${ }^{2}$ LEGMA-DQB, Departamento de Química Biológica, Facultad de Ciencias Exactas y Naturales, Universidad de Buenos Aires,

${ }^{3}$ IFIBYNE - CONICET, ARGENTINA,

${ }^{4}$ CEFYBO-CONICET, Facultad de Medicina, Universidad de Buenos Aires,

${ }^{5}$ DBMS, Ontario Veterinary College, University of Guelph, Canada,

${ }^{6}$ Program in Gene Function and Expression, University of Massachusetts Medical School, Worcester MA.

${ }^{7}$ CINIBA, Facultad de Ciencias Médicas, Universidad Nacional de La Plata, UNLP

*, $\neq$ Contributed equally to this study.

† Corresponding Author 
Alternative polyA of Itgb1 in mammary gland

\begin{abstract}
Integrins are heterodimeric cell surface adhesion receptors that play a critical role in tissue development. Characterization of the full length mRNA encoding the $\beta 1$ subunit (Itgb1) revealed an alternative functional cleavage and polyadenylation site that yields a new Itgb1 mRNA isoform, 578bp shorter than that previously reported. Using a variety of experimental and bioinformatic approaches, we found that the two Itgb1 isoforms are expressed at different levels in a variety of mouse tissues, including the mammary gland, where they are differentially regulated at successive developmental stages. The longer mRNA species is privileged during lactation, while the shorter is induced after weaning. In 3D cultures, where expression of Itgb1 protein is required for normal formation of acini, experimental blockade of the longer isoform induced enhanced expression of the shorter species that allowed normal morphological mammary differentiation. The short isoform lacks AU-rich motifs and miRNA target sequences that are potentially implicated in the regulation of mRNA stability and translation efficiency. We further determined that the AU binding protein HuR appears to selectively stabilize the longer isoform in the mammary gland. In summary, our data identify a new regulatory instance involved in the fine-tuning of Itgb1 expression during mammary gland development and function.
\end{abstract}


Alternative polyA of Itgb1 in mammary gland

\section{Introduction}

The extracellular matrix (ECM) is a complex mixture of molecules that surrounds cells providing support and conveying a variety of signals. Cell adhesion to the ECM is mediated largely by integrins, which cluster with numerous cytoskeletal and signaling proteins at focal adhesions and focal complexes [1]. Integrins are heterodimers that control many signaling events, which are critical for cell survival, growth, and gene expression regulation $[1,2]$. In mammals, the integrin family contains $18 \beta$ and $8 \alpha$ subunits that form 24 distinct receptors with specific tissue distribution and function as shown by both their specificity for ECM ligands and knockout mouse phenotypes [2].

The integrin $\beta 1$ subunit (Itgb1) is found in 12 different integrins that bind to ECM protein ligands, namely collagen, laminin, fibronectin, tenascin $\mathrm{C}$ and vitronectin $[3,4]$. $\beta 1$ integrins are expressed in a wide variety of tissues. They are critical for the induction and maintenance of cell differentiation and are involved in tissue homeostasis and diverse physiological functions [3]. Knock-out studies in mouse models have revealed an essential role for $\beta 1$ integrin in early development since genetic deletion resulted in embryonic lethality shortly after implantation $[5,6$, 7].

In the mammary gland, interaction between epithelial cells and the ECM is required for tissue development and function. Particularly, $\beta 1$ integrins play a critical role in the maintenance of mammary tissue structure and function [3]. These molecules are essential for control of cell survival and proliferation during mammary gland development, as well as for mammary epithelial cell differentiation $[8,9,10]$.

By alternative mRNA processing (as inclusion or exclusion of specific exons, poly(A) tail length, etc.) transcripts with different characteristics are generated from the same gene. Different processing alternatives may influence localization, stability, transport and translation efficiency of the mRNAs. Such differences are likely to be highly influential in cell fate changes that impinge on tissue and organism development $[11,12]$. Through alternative polyadenylation, a specific transcript has the potential to be cleaved and processed at alternative sites with the addition of a poly(A) tail (pA or poly(A) sites), resulting in mature mRNAs with 3'UTRs of different lengths. These non-coding regions influence both translation efficiency and mRNA stability. In some cases by the binding of specific proteins to well defined AU-rich sequences. These proteins are known as AUBPs (for AU-rich Binding Proteins). HuR, AUF1, KSRP and TTP are major representative members of this family [13].

In the present study, we characterized the Itgb1 3'UTR and identified an alternative polyA site that, when utilized, results in a shorter Itgb1 mRNA isoform than that reported previously. This new isoform appears to lack functional regulatory sequences that modulate Itgbl mRNA stability and translational efficiency in mammary cells. 
Alternative polyA of Itgb1 in mammary gland

\section{Materials and Methods}

\section{Cell Culture}

The HC11 cell line, derived from pregnant BALB/c mouse mammary glands, was maintained as previously described [14]. HeLaTetOff cells (Clontech, Madison, WI) were maintained in Dulbecco's modified Eagle's medium high glucose (DMEM) (Invitrogen, Grand Island, NY), with L-glutamine and sodium pyruvate supplemented with 10\% fetal bovine serum and penicillin/streptomycin/amphotericin B (Invitro-gen, Grand Island, NY).

\section{Cell Differentiation and Cell Stretching}

HC11 cell differentiation was induced as previously described [14]. To perform stretching assays, laboratory made silicone membranes, coated with collagen type I, were placed inside Petri dishes $(60 \mathrm{~mm}$ diameter $)$. Cells were plated at a density of $7 \times 105$ cells/dish. After attachment, they were grown for approximately $48 \mathrm{~h}$, until the cell culture became confluent. Membranes with the attached cells were removed from the culture dishes and carefully placed inside the stretching device after which the unit was assembled as previously described [15]. Prewarmed serum-free cultura medium was added on top of the cells. Membranes were exposed to sustained radial strain of $20 \%$ for different time intervals ( $3 \mathrm{~h}$ to $5 \mathrm{~h}$ ). Afterwards, mRNA extraction protocols were carried out as described below.

\section{Transient Transfections}

HC11 and HeLa-TetOff cells were plated in complete media and were allowed to grow overnight to $70-80 \%$ confluence. The cells were transfected using linear polyethylenimine/PEI (Polysciences, Warrington, PA) with a protocol adjusted for each cell line: 2,4 or $10 \mu \mathrm{g}$ of total DNA, for 6-well plates, 6-cm or $10-\mathrm{cm}$ dishes respectively, were incubated in the presence of PEI $(1 \mu \mathrm{g} / \mathrm{uL} \mathrm{pH} 7,2)$, in a 3:1 ratio (PEI:DNA) for both cell lines, in OptiMEM media (Invitrogen, Grand Island, NY) for 10 minutes. RPMI or DMEM media, for HC11 and HeLaTetOff cells respectively, without serum was then added to the mixture. These complexes were added to the cell plates that were previously washed 3 times with PBS 1X. After 3 to $6 \mathrm{~h}$ cells were washed with PBS $1 \mathrm{X}$ and the transfection mixture was changed to growth medium.

\section{DNA Constructs}

The PCR fragment corresponding to the 3'UTR region present only in the short isoform (Itgb1 3'UTR Short) (NM_010578, bases 2719 to 3232) and the fragment corresponding to the differential region between the long and short isoforms (Itgb1 3'UTR D) (NM_010578, bases 3232 to 3810) were cloned downstream of the Luciferase gene in the pGL3 control plasmid using the Xba I restriction site. In addition, the Itgb1 3'UTR "D" fragment was cloned into the pTRE2hygLuc plasmid (Clontech, Madison, WI) using the Nhe I and Sal I restriction sites (pLuc Itgb1 3'UTR D). A fragment containing the Itgb1 ARE1 region, plus 50 bp upstream and $50 \mathrm{bp}$ downstream, was cloned using the CloneJET ${ }^{\mathrm{TM}}$ PCR Cloning Kit from 
Alternative polyA of Itgb1 in mammary gland

Fermentas, to prepare probes for the RNA EMSA assays (pJET-Itgb1ARE1) by in vitro transcription. The same sequence was inserted in an inverted orientation in order to generate a suitable control for specificity (pJET-Itgb1ERA1). The cDNA of mouse HuR was subcloned into pCEFL-HA and pGEX4T3 (María Sol Degese et al, manuscript in preparation). The PAS1 region, flanked by a 200 bp sequence (00 bp upstream and $100 \mathrm{bp}$ downstream), was cloned into the pRiG vector using the Xho I and BamHI restriction sites to build pRiG-Itgb1.pA1. Plasmids pRiG and pRiG77s.AE were kindly provided by Dr. Bin Tian (Univ. of New Jersey). Point mutations in the PAS1 region were introduced using the QuickChangeII site-Directed Mutagenesis Kit (Stratagene). The wild type PAS1 sequence (ATTAAAATAAA) was therefore mutated to the sequence AGTAAAAGGAA (pRiG-Itgb1.pA1mut).

\section{Real-time PCR}

Total cellular RNA was isolated using TriZol (Invitrogen, Grand Island, NY) or TriReagent (Genbiotech, Buenos Aires, Argentina) using the manufacturer's protocols. mRNAs were reversetranscribed using oligo-dT primers (Promega, Madison, WI). Real-time quantitative PCR was performed using a Stratagene MX 3000P QPCR with SYBR green (Roche, Basel, Switzerland) detection. For the real time PCR $2 \mu \mathrm{g}$ of total RNA was subjected to reverse transcription. A 1 in 10 dilution of all samples was then used to measure the expression of the endogenous Itgb1 isoforms relative to GAPDH expression. The following primers were utilized for the different Itgb1 mRNA isoforms: Itgb1-T forward (5'-CGTCGCAGCATATGAACC3') reverse (5'-AACAAAGCCACCAGCAAGG-3'); Itgb1-S forward (5'GCAGCATCTGAACCAT-GAC-3'), reverse (5'-TTTTTTTTTTTTTTTTTGAAC3'); Itgb1-L forward (5'-AAACCTGTGT-GCCATTGTG-3'), reverse (5'GCCTTTATGTGAATAGTGCTAAG-3'). HuR: Forward (5'TGGGCTACGGTTTTGTG-3'), Reverse (5'-GGACCCTGGAGTTGATGATT-3').

\section{Western blotting}

The protein extracts were collected from mammary gland tissues and cell lysates and homogenized in RIPA buffer $(50 \mathrm{mM}$ Tris- $\mathrm{HCl} \mathrm{pH} 8.0,150 \mathrm{mM} \mathrm{NaCl}$, $1 \%$ NP40, $0.5 \%$ sodium deoxycholate, $0.1 \%$ SDS) with protease and phosphatase inhibitors (Pierce, Rockford, IL). After heat denaturation, equal amounts of protein lysates, as previously determined by Bradford's technique, were separated on $8 \%$ SDS-polyacrylamide gels and transferred to nitrocellulose membranes. The blots were blocked in 5\% non-fat dry milk for 1 hour and incubated overnight with anti-Itgb1 (1:600, sc-8978 M-106 Santa Cruz), anti-HuR (1:1000, sc-5261 3A2 Santa Cruz), anti-ciclin D1 (1:600, sc-8396 A-12 Santa Cruz) or anti-actin (1:2000, sc-1616 I-19 Santa Cruz) at $4^{\circ} \mathrm{C}$. After incubation with infrared-conjugated secondary antibodies, the membrane was scanned in an Odyssey SA scanner (LICOR Biosciences, Lincoln, $\mathrm{NE}$ ), and band intensities were quantified using the Odyssey software.

\section{Morphogenesis assay}

HC11 cells were treated with trypsin and resuspended in growth medium. The cells were pelleted and resuspended in assay medium (RPMI supplemented with 2\% 
Alternative polyA of Itgb1 in mammary gland

FBS, $2 \mathrm{mM}$ L-glutamine, $2.5 \mathrm{ng} / \mathrm{ml} \mathrm{EGF}$ and $5 \mathrm{~g} / \mathrm{ml}$ insulin) at a concentration of 25,000 cells $/ \mathrm{ml}$. Eight chambered RS glass slides (Nalgene) were coated with $40 \mathrm{uL}$ Matrigel basement membrane matrix per well and left to solidify for $15 \mathrm{~min}$. The cells were mixed 1:1 with assay medium containing 4\% Matrigel, and $400 \mathrm{uL}$ was added to each chamber of the Matrigel-coated eight-chambered slide. Assay medium was replaced every $4 \mathrm{~d}$.

\section{Indirect immunofluorescence and image acquisition}

The following primary antibodies were used for indirect immunofluorescent detection of specific antigens: anti-cleaved caspase 3 (Asp175; Cell Signaling) and Anti-Laminin 5 antibody (ab78286; Abcam). All first antibodies were used at a dilution of 1:200. Secondary antibodies were as follows: anti-mouse or anti-rabbit coupled with Alexa Fluorescent dyes. All secondary antibodies were used at a dilution of 1:500. Nuclei were stained with DAPI. Confocal analyses were performed using confocal Olympus Fluoview FV 1000 microscope.

\section{shRNA knockdown of Itgb1 expression}

Itgb1 mouse shRNA (OligoIDs: V2LMM_39157, V3LMM_429938, V2LMM_188403) and Non-silencing-GIPZ lentiviral shRNAmir control (R्̈HS4348) clones were obtained in pGIPZ-GFP vectors (Thermo Scientific Open Biosystems, Huntsville, AL) and transfected into HC11 cells using PEI. Four stable cell lines were generated: Sh Itgb1-T, Sh Itgb1-L and Sh control. The knockdown of Itgb1 expression was analyzed by western blot.

\section{siRNA Knockdown of HuR expression}

HuR siRNA and AllStars Negative Control siRNA (QIAGEN) were introduced in HC11 cultured cells by cell transfection using Lipofectamine 2000 (Invitrogen) according to the manufacturer's instructions at a concentration of $40 \mathrm{nM}$. The knockdown effects were analyzed 3 days after transfection by RT-qPCR and western blot as shown.

\section{Analysis of Reporter and Endogenous mRNA decay by Real Time PCR}

To measure reporter mRNA decay, HeLaTetOff cells (Clontech, Madison, WI) were transiently transfected using PEI reagent with each reporter construct: pLuc Itgb1 3'UTR D and pCEFL-HA-HuR. This cell line expresses the tTAS2 transactivator that induces transcription in the absence of tetracycline or its analog doxycycline. After transfection, cells were incubated in tetracycline free media for $24 \mathrm{~h}$, then tetracycline was added to a final concentration of $1 \mu \mathrm{g} / \mu \mathrm{L}$, for a period from 
Alternative polyA of Itgb1 in mammary gland

0 to $5 \mathrm{~h}$. After this, cells were lysed and RNA was isolated following the manufacturer's protocols as described above. $2 \mu \mathrm{g}$ of total RNA was treated with RQ1 RNase free DNase (Promega, Madison, WI) for 15 minutes, followed by first strand cDNA synthesis using MMLV reverse transcriptase (Promega, Madison, WI), RNasin (Promega, Madison, WI) and OligodT (Biodynamics, Argentina). Luciferase mRNA was quantified by qPCR using the following primer set: Forward (5'CCGCCGTTGTTGTTTTG- 3'); Reverse (5'- ACACAACTCCTCCGCGC-3'). Luciferase mRNA expression was normalized against the hygromycin resistance gene product which is present in the reporter plasmid. Primer sequences were: Forward (5'GGAATCCCCGAACATCG-3'); Reverse (5'-GCAGACGCGCTACTTCG-3'). To measure endogenous Itgb $1 \mathrm{mRNA}$, proliferating or differentiated $\mathrm{HC} 11$ cells were incubated with Actinomycin D to a final concentration of $5 \mu \mathrm{g} / \mathrm{mL}$, for a period from 0 to $5 \mathrm{~h}$ cells and lysates were then analysed as described above.

\section{RNA Electrophoretic Mobility Shift Assays}

RNA probes were synthesized with a T7 RNA transcription Kit (Promega, Madison, WI) using the pJETItgb1ARE1 and pJET-ItgblERA1 plasmids. For radioactive probes $[\alpha-32 \mathrm{P}] \mathrm{rUTP}$ (NEN Perkin Elmer, Waltham, MA), was added. Total extracts were obtained from HC11 cells that had been plated on 6 well plates using growth conditions and from tissue samples derived from normal mouse mammary gland. Protocols for extraction and incubation have been previously described (16). Cells were washed with cold PBS 1X, and lysed with Lysing Buffer: 10mM HEPES pH 7.6, 3mM MgCl2, $40 \mathrm{mM} \mathrm{KCl,} 2 \mathrm{mM}$ DTT, $5 \%$ glycerol, $0.5 \%$ igepal, $1 \mathrm{mM}$ phenylmethylsulfonyl fluoride (PMSF), $10 \mathrm{mM} \mathrm{NaF}, 1 \mathrm{mM}$ sodium orthovanadate, $10 \mathrm{mM}$ betaglycerophosphate, and protease inhibitor cocktail (Sigma). After centrifugation $\left(5\right.$ minutes, $14000 \mathrm{rpm}, 4^{\circ} \mathrm{C}$ ) the protein concentration of each sample was measured using Bradford's technique. $5 \mu \mathrm{g}$ of total protein was preincubated with BSA (final concentration $500 \mathrm{ng} / \mu \mathrm{L})$, tRNA $(125 \mathrm{ng} / \mu \mathrm{L})$ and heparin $(10 \mathrm{ng} / \mathrm{uL})$ for 5 minutes at room temperature (RT). 2-4 $\mathrm{ng}$ of radioactive probe was added to the mix followed by incubation at RT for 20 minutes. Finally single stranded (unbound) RNA was digested with 0.6 units of Rnase T1 incubated for 10 minutes RT. The ribonucleocomplexes were resolved on pre-run (20 min), 6\% non-denaturing polyacrylamide gels with a TBE buffer system for $1.5 \mathrm{~h}$.

\section{Bacterial Expression of GST Fusion Proteins}

The BL21 pLys E. coli strain was transformed with a construct based on the vector pGEX-4T3 encoding the fusión protein GST-mHuR WT by insertion of a DNA fragment containing the HuR open reading frame. Bacteria were grown in 500 $\mathrm{mL}$ of LB medium until the optical density was 0.5 , at which time isopropyl beta-D1-thiogalactopyranoside ( $1 \mathrm{mM}$ final) was added for $3 \mathrm{~h}$. Bacterial pellets were collected by centrifugation at $3000 \mathrm{~g}$ for $30 \mathrm{~min}$ and resuspended in $10 \mathrm{ml}$ of PBS, $1 \%$ Triton X-100, $1 \mathrm{mM}$ EDTA, $2 \mathrm{~g} / \mathrm{ml}$ aprotinin, $2 \mathrm{~g} / \mathrm{mLleupeptin,} \mathrm{and} 1 \mathrm{mM}$ PMSF. Suspensions were then sonicated and debris removed by centrifugation at $10,000 \mathrm{x} \mathrm{g}$ for $15 \mathrm{~min}$. Supernatants were mixed with $300 \mu \mathrm{L}$ of glutathione-agarose beads (Amersham Biosciences, Piscataway, NJ) and centrifuged at $3000 \mathrm{x}$ g for 5 minutes. The pellet was washed three times with PBS, 1\% Triton X-100, 1mM EDTA, 
Alternative polyA of Itgb1 in mammary gland

protease inhibitor cocktail (Sigma-Aldrich, Saint Louis, MO), and 1mM PMSF and then twice with PBS, protease inhibitor cocktail, and $1 \mathrm{mM}$ PMSF. Finally, purified fusion proteins were eluted in $50 \mathrm{mM}$ Tris, $10 \mathrm{mM}$ glutathione, protease inhibitor cocktail, and $1 \mathrm{mM}$ PMSF.

\section{Luciferase Reporter Assays}

HC11 cells were transiently transfected with each reporter construct using PEI. The amount of luciferase mRNA was quantified using the primers described above, and normalized relative to GAPDH: Forward (5'AAGAaGGTGGTGAAGCAGGCATC-3'), and Reverse (5'CGAAGGTGGAAGAGTGGGAG-TTG-3'). For luciferase activity assays, cells were seeded on 6-well or 12-well dishes and transfected with $0.1 \mu \mathrm{g}$ of luciferase reporter vector and a vector that constitutively expresses betagalactosidase. Cells were lysed in passive lysis buffer (Promega, Madison, WI) 24h post-transfection. Cell lysates were incubated with luciferin reagent (luciferase Reporter System - Promega, Madison, WI). Data was normalized for efficiency of DNA transfection by measuring betagalactosidase activity.

\section{Animal treatments}

$\mathrm{BALB} / \mathrm{c}$ mice were maintained in a pathogen-free, temperature controlled environment on a " $12 \mathrm{~h}$ light $/ 12 \mathrm{~h}$ dark" cycle and given sterilized laboratory chow and water ad libitum in accordance with the standard international animal care protocols (Canadian Council of Animal Care's 1980 Guide to the Care and Use of Experimental Animals). Animal procedures were carried out at the Animal Facility of the School of Sciences of the University of Buenos Aires (Facultad de Ciencias Exactas y Naturales, Universidad de Buenos Aires) following the guidelines provided by the University of Buenos Aires Institutional Animal Care and Use Committee, which oversees the university's animal care and use program and is responsible for reviewing all animal use protocols (U.S. Public Health Service Approved Animal Welfare Assurance: A5801-01). The fourth mammary glands were aseptically removed from virgin, pregnant, lactating ( $7 \mathrm{~d}$ after delivery) and involuting (24h; $48 \mathrm{~h}$ and $72 \mathrm{~h}$ after weaning (pups were removed after 5-7 d of nursing)) females.

\section{FACS analysis}

HC11 cells grown in 6-well plates were transfected with different pRiG derived vectors. Cells were harvested $48 \mathrm{~h}$ after transfection and were subjected to fluorescent activated cell sorting (FACS) analysis. Briefly, cells were treated with $0.25 \%$ trypsin solution to release them from culture dishes. Green and Red fluorescence were read at $530 \mathrm{~nm}$ and $585 \mathrm{~nm}$, respectively, using the BD FACScalibur system (BD Biosciences, San José, CA). Transfection with a plasmid expressing EGFP alone was used to adjust the green fluorescence channel. The $\mathrm{R}$ program was used to draw the cumulative fraction curves, using codes for plotting as previously described [17].

\section{Rapid Amplification of cDNA 3' Ends (3'RACE-PCR)}


Alternative polyA of Itgb1 in mammary gland

The first strand cDNA synthesis was performed for $2 \mathrm{~h}$ at $37^{\circ} \mathrm{C}$ on a reaction mixture containing $3 \mu \mathrm{g}$ of total RNA, 10 units of RNAsin (Promega Biotech, Madison, WI), 100U of reverse transcriptase (RT) (SuperScript, Gibco, Grand Island, $\mathrm{NY}$ ), $1 \mathrm{mM}$ of DTT, 1 X RT Buffer, $0.5 \mathrm{mM}$ of dNTPs and $1 \mu \mathrm{g} / \mu \mathrm{L}$ of the adaptor primer Ad-(dT)15 (5'-GTCGCGAATTCGTCGACGCG-(dT15)-3'). PCR reactions were carried out using a specific forward primer for Itgbl (Ex17: 5'AGAAAATGAATGCCAAGTGGG-3') and the reverse primer Ad-(dT)15 as outlined in Figure 2. The products were analyzed on $0.7 \%$ agarose gels stained with ethidium bromide. After several restrictive PCR conditions, the fragments of interest were purified using the QIAquick Gel Extraction Kit (Qiagen, Germany), directly ligated into the pGEMT Easy Vector System (Promega, Madison, WI), and sequenced. The identities of the fragments were analyzed using public databases.

\section{EST and SAGE database mining}

Mouse ESTs corresponding to the Itgb1 transcript (Mm.263396) were downloaded from the UniGene database (http://www.ncbi.nlm. nih.gov/unigene) and comprised a total of 771 EST sequences. Two-hundred and eighty-three 3'-read ESTs with polyadenylation sites were extracted and aligned against the mouse Itgbl gene using the BLASTN algorithm at NCBI. The EST end positions were mapped back to the genome location (NCBI37/mm9 assembly) using the UCSC genome browser resource (http://genome. ucsc.edu). To perform a comparative analysis of the mouse Itgb1 variants expressed in different mouse tissues, we analyzed $269 \mathrm{mSAGE}$ libraries downloaded from the Cancer Genome Anatomy Project - SAGE Genie database (http://cgap.nci.nih.gov/SAGE/). SAGE tag counts were normalized to 200,000 tags per library according to the total tag count in each library. Three reliable SAGE tags were found for Itgbl transcripts: (5'-CCAGGGACTG-3') (2877bp relative to the starting position of the NM_010578.2 sequence), (5'-ACTGATTTTC3') (3051bp), and (5'-CTTGCTCTGT-3') (3706bp), among which (5'CTTGCTCTGT-3') corresponded to the Itgb1-L variant, and (5'-CCAGGGACTG$3^{\prime}$ ) and (5'ACTGATTTTC-3') corresponded to the Itgb1-S variant. Expression values for both Itgb1-S tags were combined for statistical analysis. MultiExperiment Viewer software (MeVv4.7) was used to enable the visualization and illustration of our analyses [18]. This tool was employed for normalization and hierarchical clustering analysis of the SAGE data. 
Alternative polyA of Itgb1 in mammary gland

\section{Results}

\section{Alternative cleavage and polyadenylation sites in the Itgb1 gene results in the expression of two mRNA isoforms}

Sequence analysis of the mouse Itgbl gene identified two distinct poly(A) consensus sequences located in the 3'UTR which suggested the existence of alternative cleavage and polyadenylation sites. Our observation of 3'-read EST sequences confirmed the existence of two functional cleavage and polyadenylation sites in the Itgbl gene and two clusters corresponding to the alternative polyA sites were identified (Figure 1A). It was determined that the longer isoform (Itgb1-L) represented approximately 92\% (260 out of 283) and the Itgb1-S isoform 8\% (23 out of 283) of the 3'-read Itgb1 EST sequences in the mouse. To verify the presence of the Itgb1 species in a greater variety of tissues, the 3'-read ESTs were further analyzed using Unigene annotations. The Itgb1-L variant predominated in brain, eye and heart libraries, where it represented $95-100 \%$ of total Itgb1 transcripts. In contrast, in the mammary gland, ovary and pancreas there was significant enrichment of the Itgb1-S species, which constituted $25-40 \%$ of all Itgb1 ESTs (Figure 1B). Similarly, using a publicly-available dataset of 269 mouse SAGE libraries, we determined that the Itgb1-L transcript is expressed at higher levels than the Itgb1-S in almost all libraries analyzed. Again, Itgb1-S was detected at consistently higher levels in mammary gland, urogenital and vascular endothelial tissues than in brain and eye (retina) tissues (Figures 1B and 1C).

To experimentally confirm the presence of these alternative Itgb1 isoforms by PCR analysis, we designed specific primers that bind to the last exon of the mouse Itgb1 gene, encompassing the identified distinct poly(A) consensus sequences (PAS1 and PAS2) and potential cleavage sites located in the 3'UTR (Figure 2A and 2B). The mammary gland was chosen to study the expression of the Itgb1 isoforms, because it allowed us to determine whether they can be developmentally regulated in the adult animal. Therefore, mouse mammary glands at different stages of development, as well as MMTV-induced mouse mammary tumors [19], were used as sources of mRNA. The 3'UTR regions of Itgb1 transcripts were isolated by 3'RACE PCR, producing $1400 \mathrm{bp}$ and $750 \mathrm{bp}$ amplicons (Figure $2 \mathrm{~B}$ and $2 \mathrm{C}$ ). Sequence analysis of the fragments confirmed the presence of the potentially functional poly(A) sites (Figure 2D). The shortest 3'UTR corresponded to Itgb1-S and was composed of 578 nucleotides, while the longer Itgb1-L 3'UTR was 1110 nucleotides in length. Interestingly, Itgb1-L sequence showed two ARE class I sequences, ATTTA (ARE1 and ARE2, in Figure 2D and 2E) that were absent in the Itgb1-S variant.

\section{The proximal pA1 site in the 3'UTR of Itgb1 is fully functional}

To further confirm the functionality of the Itgb1 proximal polyadenylation site (pA1) in mammary cells, the pRiG vector was used. This reporter expresses a bicistronic transcript for two fluorescent proteins (RFP and EGFP) and includes a polylinker between the two open Reading frames (ORFs). When a functional site for cleavage and polyadenylation is inserted between the ORFs, the proportion of 
Alternative polyA of Itgb1 in mammary gland

expressed fluorescent proteins is altered. Then, mouse mammary HC11 cells were transfected with the different pRiG variants. In one of them, the $200 \mathrm{bp}$ region surrounding PAS1 (see Figure 2D and 2E) wild type and mutated were introduced into the cloning site of this vector (Figure 3A, pRiGItgb1.pA1 and pRiG-Itgb1.pA1 mut) and expression of the fluorescent proteins were analyzed by flow cytometry. Expression of the pA1 construct ( $p$ RiG-Itgb1.pAl) showed a higher intensity of red fluorescence (RFP) than the empty vector ( $\mathrm{pRiG}$ ) or a pRiG construct containing a mutated pA1 element (Figure 3B). The EGFP/RFP cumulative fraction (Fig 3B lower panel) indicates that expression of pRiGItgb1.pA1 led to significantly lower EGFP/RFP ratios ( $\mathrm{P}<2.2 \mathrm{E}-16$, Kolmogorov-Smirnov test), which demonstrates the functionality of the proximal Itgbl cleavage site. Furthermore, the ratio between the percentage of events shown in the upper left quadrant (mostly red) to the lower right quadrant (mostly green) from four independent FACS analyses (Figure 3C) clearly shows that pA1 sequences are recognized by the cleavage and polyadenylation machinery of mammary epithelial cells, resulting in the production of a functional mRNA species.

\section{Expression pattern of Itgb1 isoforms during differentiation and involution of the mouse mammary gland}

Analysis of Itgb1 protein expression in the mouse mammary gland (epithelium and stroma) at successive developmental stages showed an increase during pregnancy and lactation (Figure 4A). On the other hand, RT-qPCR assays revealed a reduction of Itgb1 mRNA levels during lactation, as previously reported [20] (Figure 4B). Then, using primers specially designed to amplify each Itgb1 mRNA isoform (see figures $2 \mathrm{D}$ and $2 \mathrm{E}$ ), we proceeded to evaluate whether each species showed a differential expression pattern during mammary gland differentiation and involution in adult female mice (Figures 4C and 4D). Here, is important to point out that despite the low complexity of the Itgb1-S reverse primer, amplification of this template resulted in a single specific product (data not shown). The analysis revealed that the ratio of mRNA expression for each Itgbl isoform changes at different stages of development in mammary tissue. From virgin to lactating glands Itgb1-L mRNA levels show little variation, while Itgb1-S decreases to undetectable levels (Figre 4C). Alternatively, a strong increase of Itgb1-S expression was detected at $72 \mathrm{~h}$ of involution (Figure 4C and D). Interestingly, the application of a stretching protocol, which triggers involution-associated molecular events in HC11 mammary cells [15], also induced Itgb1-S and inhibited Itgb1-L expression in culture (Figure 4E). Taken together, these results suggest that although total Itgb1 mRNA expression might be reduced in the differentiated mammary gland, the longer isoform would be stabilized and efficiently translated during lactation.

\section{Biological relevance of the Itgb1-S isoform.}

The data reported above suggested to us that the Itgb1-L isoform prevails in the lactating mammary gland. In order to test whether expression of this species is required for mammary epithelial cell differentiation, we used a combination of 
Alternative polyA of Itgb1 in mammary gland

shRNA plasmids to block the expression of the Itgb1-L isoform specifically, or both species, Itgb1-L and $\mathrm{S}$, together. The shItgb1-L was designed to bind a region between pA1 and pA2 (see Figure 5A) and, therefore, to reduce Itgb1L mRNA exclusively, without affecting Itgb1-S levels. On the other hand, shItgb1-T would bind sequences upstream to pA1, lowering the levels of both mRNA isoforms. We found that $\mathrm{HC} 11$ cells stably transfected with shItgb1-T show an important reduction of Itgb1 protein levels, while cells transfected with shItgb1-L show an intermediate level of Itgb1 protein. Interestingly, CiclinD1 levels, which are clearly reduced in cells transfected with shItgb1-T, are not affected by the shItgb1-L (Figure 5A). Analysis of mRNA expression by qPCR, show that both shRNAs have a similar detrimental effect on Itgb1-L expression; alternatively, expression of the Itgb1-S isoform is significantly increased when Itgb1-L is specifically targeted (Figure 5B). To analyze the effects of the Itgb1 shRNAs on morphological differentiation, 3D cultures of $\mathrm{HC} 11$ cells were performed by seeding the cells in Matrigel coated dishes. Figure 5C shows that the specific blockade of Itgb1L does not affect formation of acini that show characteristic features of differentiated structures including expression of cleaved caspase 3 in the interior and laminin V surrounding them (Supplementary Figure 1). Interestingly, HC11 cells transfected with shRNA-T also form acini, although they are reduced in size, number and show an altered morphology (Figure $5 \mathrm{C}$ and Supplementary Figure 1). These results indicate that Itgb1-L is not required per se for mammary gland differentiation, while a minimum of Itgb1 protein expression, which would be mostly translated from the Itgb1-S isoform in the shItgb1-L transfected cells, might be necessary for the epithelium to successfully complete morphological differentiation.

\section{Itgb1-L is specifically stabilized during mammary gland differentiation}

Figure 4 shows that Itgb1-S mRNA is significantly reduced during pregnancy and lactation, while not significant changes affected Itgb1-L levels. Therefore, we speculated that the longer isoform might be specifically stabilized during that period, allowing efficient expression of the Itgb1 protein. To examine this possibility we proceeded to analyze the stability of each Itgb1 isoform in proliferating and differentiated HC11 mammary cells [14]. Therefore, the level of both Itgb1 mRNA endogenous species remaining at specific times after the addition of the transcriptional inhibitor Actinomycin D was analyzed by qRT PCR in mRNA isolated from proliferating or differentiated cells. Figure 6A demonstrates that Itgb1-L mRNA is more stable in differentiated than in proliferating $\mathrm{HC} 11$ cells. In contrast, Itgb1-S was unstable under both conditions (Figure 6B). Figures $2 \mathrm{D}$ and $2 \mathrm{E}$ indicate that Itgb1-L includes ARE sequences that are not present in the shorter isoform. Therefore, we postulated that stabilization of the longer isoform might be due in part to the presence of AUBPs that bind specifically to those sequences. Among the wellcharacterized AUBPs, HuR has been described to have a stabilizing role on bound mRNAs [21]. Therefore, to determine whether this AUBP might stabilize Itgb1-L mRNA in the mammary gland, we first analyzed its expression profile in both proliferating and differentiated cells. We found that HuR expression levels significantly increased as measured by qPCR and Western Blot (Figures 6C and 6D, respectively). We also determined that the levels of HuR mRNA and Itgb1 protein correlated in mouse mammary samples as HuR mRNA and protein also peaked 
Alternative polyA of Itgb1 in mammary gland

during lactation (Figures $6 \mathrm{E}$ and $6 \mathrm{~F}$ ). These data suggested to us that HuR was likely to contribute to the slower decay of Itgb1-L mRNA in vivo.

\section{The AUBP HuR contributes to the stabilization of the Itgb1- $L$ isoform}

To determine whether HuR binds to ARE sequences in the Itgb1-L 3'UTR, we evaluated the effect of HuR overexpression on the stability of a luciferase reporter mRNA containing the ARE1 and ARE2 sequences derived from the Itgb1-L isoform (see Figures 2D and 2E). Importantly, this construct included only the "differential region" between the Itgb1-L and the Itgb1-S (3'UTR-D) (Figure 7A). HeLa-TetOff cells were transfected with the LUC-3'UTR-D reporter construct under the control of a tetracycline responsive promoter (Tet-off) along with either a plasmid that expressed HuR or an empty vector. Cells were then incubated with tetracycline to selectively suppress reporter gene transcription and allow the assessment of mRNA stability. Luciferase expression was analyzed at different time points by qRT-PCR. Figure 7B shows that the luciferase mRNA fell to $50 \%$ after $4 \mathrm{~h}$ when the reporter plasmid was co-transfected with an empty vector, while little mRNA decay was observed when HuR was co-expressed in the same cells. These results were consistent with HuR binding and stabilizing Itgb1-L mRNA through interactions with the AREs in the "D" region of the mRNA. To determine whether ARE1 in the Itgb1-L mRNA interacts with recombinant HuR protein or with endogenous HuR in mammary cells, RNA Electromobility Shift Assays (RNA EMSA) were performed. Figure 7C shows that a recombinant GST-HuR protein formed a complex with radiolabelled probe containing Itgb1-ARE1 sequences that migrated with decreased mobility relative to the free probe. Comparable bands were not formed after incubation of probes with GST protein alone. Proteins within HC11 cell lysates also formed complexes with the same probe and not with a labeled control probe with an antisense orientation. Protein binding to the ARE1 probe was competitively inhibited by an excess of unlabeled ARE1 probe. Preincubation of the HC11 lysates with a HuR antibody resulted in a further decrease in electrophoretic mobility (supershift) that was not observed after incubation with an antibody against GFP, suggesting the specific presence of HuR in the protein/probe complex. Protein extracts of mouse mammary glands at different developmental stages were incubated with the same probe and we observed a particular array of retarded bands that varied depending on the cell source (Figure 7D). Specific bands that correspond in position with HuR containing complexes were present in all the samples. In addition, we proceeded to specificly knockdown HuR expression with siRNA in proliferating HC11 cells. This procedure reduced HuR mRNA and protein levels by $70 \% 3$ days after transfection (Figure 7E and inset). This led to a significant reduction of remaining Itgb1-L mRNA endogenous species $3 \mathrm{~h}$ after the addition of the transcriptional inhibitor Actinomycin D (Figure 7F). Altogether the results in Figures $7 \mathrm{~B}$ to $7 \mathrm{~F}$ indicate that $\mathrm{HuR}$ is important for the stabilization of the Itgb1-L isoform in $\mathrm{HC} 11$ cells.

The $3^{3}$ UTR of the Itgb1-L isoform contains sequences that participate in regulation of translation efficiency 
Alternative polyA of Itgb1 in mammary gland

In addition to effects on mRNA stability, AUBPs bound to AREs are known to influence the translation of target mRNAs. We therefore determined whether the differential region in the 3'UTR of Itgb1-L could also modulate translation. To this end, we analyzed the translation efficiency of luciferase mRNAs under the control of a constitutive promoter and bearing different Itgb1 3'UTRs. HC11 cells were transfected either with a plasmid containing the Itgb1-S 3'UTR (3'UTR-S) or the 3'UTR-D (shown in Figure 7A). Figure 7G (upper panel) shows that Luciferase activity (ie. protein level) was almost 4 times lower in cells transfected with the construct containing the 3'UTR-S compared to cells transfected with the Luciferase3'UTR-D reporter. Surprisingly, based on the predicted differences in mRNA stability from the earlier studies, luciferase mRNA levels, measured by qPCR, were similar with both constructs (data not shown). Based on the ratio of luciferase activity to luciferase mRNA (Figure 7G lower panel) this data suggests that the sequences only present in the 3'UTR of the Itgb1-L isoform, enhance the translational efficiency of the $L U C$ gene.

\section{Discussion}

The development of multi-cellular organisms results, to a large extent, from changes in the pattern of gene expression within different constituent cells. These changes are the cellular response to environmental signals that are transduced into the cell and affect metabolism, transcription and translation among other biochemical processes. In addition to the more widely-studied mechanisms that control gene transcription, mechanisms of posttranscriptional RNA processing have increasingly been recognized as important participants in the regulation of gene expression [11, 12]. Such mechanisms include: alternative splicing, regulation of mRNA stability and alternative 3 ' end processing or polyadenylation [11, 12]. In the present study, we have identified two different mRNA transcripts encoding murine Itgbl that result from differential cleavage and polyadenylation of the transcript. Posttranscriptional processing of Itgbl transcripts results in a shorter isoform (Itgb1-S, $532 \mathrm{nt}$ long 3'UTR) than the one that had been previously-reported (Itgb1-L, $1100 \mathrm{nt}$ long 3'UTR). Here, we show that both isoforms are expressed by several mouse cell types, including epithelial cells from the mammary gland. In studies presented as supplementary data, 3 RACE PCR and sequence analysis of the human $\beta 1$-integrin revealed similar results to those obtained from the mouse, suggesting that this process is conserved from an evolutionary perspective (Supplementary Figure 2).

There are several mechanisms by which two alternative mRNA variants can be expressed from a single gene. One of them is the differential usage of an alternative cleavage and polyadenylation $(\operatorname{poly}(A))$ site. Sequence analysis of the Itgb1 3'UTR confirmed the existence of a potential poly(A) signal downstream from the stop codon, approximately half way to the previously-reported 3' end. We validated its functionality in mammary epithelial cells using a reporter system [22]. This proximal poly(A) site displayed weaker activity compared to the strong polyadenylation signal from the control gene $77 \mathrm{~s}$. AE, although remarkably stronger than the control reporter or a mutated version of this same poly(A) site. Weaker activity of the proximal site in Itgb1 compared to the distal site is consistent with the observed percentage of usage observed in the analysis of tissue libraries. 
Alternative polyA of Itgb1 in mammary gland

Functionally, integrins connect living cells with the extracellular matrix, facilitating physical integration with the cellular entourage and signaling interactions. Specifically, Itgb1 expression is required for the maintenance of functional mammary stem cells, allowing mammary morphogenesis and segregation of the major mammary cell compartments $[23,24]$. This protein is also required for mammary differentiation, since inhibition of alveologenesis and lactation were reported when conditional deletion of Itgb1 was induced by Cre recombinase expression in the luminal cell population [9, 10]. On the other hand, tissue microarray analysis of Itgb1 protein expression in invasive breast cancer tissues revealed a trend toward high protein levels being indicative of poor prognosis [25]. These facts support the hypothesis that Itgb1 expression needs to be tightly controlled and finely tuned to allow differentiation and function of the mammary epithelium without facilitating cancer development. The data reported herein suggest that alternative cleavage and polyadenylation that let differential use of 3'UTR regions controlling mRNA stability and translatability might facilitate the precise control of Itbg1 protein levels in this tissue.

In this study, we report that the mammary gland expresses both Itgblisoforms although in the lactating gland the levels of Itgb1-S decrease to become undetectable by RT-qPCR. Possibly, this reduction is associated to the relative transcription inhibition of Itgb1, among other genes as already reported [26], during lactation. However, as mentioned above, other authors and our own data have shown the necessity of Itgb1 protein expression for functional and morphological differentiation of the mammary epithelium. When transcription of this gene is low, stabilization of the more translatable Itgb1-L isoform would help to fulfill the protein requirement. Then, after weaning, there appears to be a higher activity of the alternative, proximal, poly(A) site, or a selective destabilization of the Itgb1-L isoform. The Itgb1-S isoform lacks two AU-rich sequence motifs (AREs) implicated in the regulation of mRNA stability and translation [21]. The presence of AREs in the differential segment suggested to us that Itgb1-L and Itgb1-S mRNA stability could be differently regulated and we found that Itgb1-L mRNA is more stable in differentiated than in proliferating $\mathrm{HC} 11$ cells while Itgb1-S stability was similar under both conditions. In addition, we determined that the differential 3'UTR segment (3'UTR-D) only present in the Itgb1-L isoform confers instability to an engineered luciferase reporter construct. These data suggest that, during lactogenic differentiation, the mammary cell environment could induce stabilization of the otherwise unstable Itgb1-L isoform, probably through the selective expression of specific ARE binding proteins, collectively known as AUBPs.

Our results indicate that HuR, which has been described to have a stabilizing role on bound mRNAs [21], would be at least one of the AUBPs involved in the regulation of Itgb1 mRNA stability in the mammary gland. We have found that HuR protein and mRNA expression profile in this tissue is consistent with the stabilization of endogenous Itgb1-L mRNAs during pregnancy and lactation. In addition, HuR over-expression increases mRNA stability to a reporter transcript containing the Itgb1 3'UTR distal region and HuR knockdown using siRNA in proliferating HC11 cells decreases the stability of Itgb1-L mRNA HuR protein effectively binds a radiolabeled 
Alternative polyA of Itgb1 in mammary gland

probe that contains approximately 100 bases from Itgb1-L 3'UTR. Interestingly, it has been already reported that Itgb1 is a HuR target in T cells [27]. Our mobility shift assays also show that HuR-mRNA interactions exist in every stage of mammary gland development. However, the observed bands differ substantially from one stage to another, suggesting that HuR may be part of different specific protein complexes with a dynamic composition along the timeline of mammary gland differentiation and remodeling. Therefore, the reported HuR necessity for maintaining mammary cell proliferation and polarity [28] might be at least partially due to Itgb1-L stabilization. Here, we also show that basal levels of Itgb1 protein are required to form normal mammary acini in 3D cultures. When expression of the Itgb1-L isoform is blocked, spontaneous induction of Itb1-S mRNA species would allow some expression of the Itgb1 protein and normal development of the acini is achieved (see Figure 5). On the other hand, in the lactating epithelium in which transcription of Itgbl gene is somehow restricted and Itgb1-S levels are undetectable (see Figure 4), Itgb1 protein expression would be maintained by stabilization (see Figure 6A) and efficient translation (see Figure 7G) of the Itgb1-L mRNA isoform.

In addition to AUBPs, different studies have shown that Itgb1 is targeted by a number of distinct miRNAs including miR-29 [29], miR-183 [30] and miR-124 [31] that directly regulate its expression. Interestingly, miR-183 and miR-29 binding sites are present exclusively in the Itgb1-L isoform. In the mammary gland, low relative expression of miR-29 has been reported during lactation and high expression during late involution [32], correlating inversely with the expression levels of Itgb1-L as shown here and supporting a role for miR-29 in the degradation of the Itgb1-L mRNA isoform. Antagonism between HuR and miRNAs that promote mRNA decay (such as miR-16), have been reported for the regulation of COX-2 mRNA levels [33]. Future studies should address whether an analogous relationship links miR-29 and HuR at the Itgb1-L mRNA. Interestingly, miR recognition sites are conserved in human Itgb1 3'UTR (Supplementary Figure 3) supporting their relevance in the expression regulation of this gene in multiple mammalian species.

It has only been recently appreciated that more than half of the genes in the human genome are alternatively polyadenylated [34]. The relationship between signals that change the cell commitment, the corresponding signaling pathways and activity of gene promoters has already been well documented. In addition, solid links have been established for promoter activity and RNA processing events [35]. In particular, it has been shown that the regulation of poly(A) site choice is coupled to transcriptional activity and results in preferential expression of long 3'UTR isoforms when gene expression is low and short 3'UTR isoforms when gene expression is high [36]. As shown here, poly(A) site choice for Itgb1 might contribute to the "finetuning" of gene expression with developmental consequences [37]. A deeper understanding of alternative polyadenylation as a mechanism for transcript diversity and concomitant gene expression regulation with biological consequences is warranted and the data presented here regarding Itgb1 in the mammary gland provides a relevant model with which those studies could be pursued. 
Alternative polyA of Itgb1 in mammary gland

To precisely regulate gene expression, achieve tissue remodeling and successful development, different cells must develop the ability to selectively respond to signals such as growth factors, hormones and/or ECM ligands in a timely manner. Itgb1 plays a critical role in the relationship of cells with the extracellular matrix and its expression must therefore be tightly regulated. In summary, our study strongly suggests that alternative mRNA polyadenylation represents one important mechanism through which cell type and tissue-specific gene regulation is achieved for this gene. Two different Itgb1 3'-isoforms produced by selective utilization of the first or second PAS and poly(A) sites are reported here for the first time. These alternative mRNA isoforms appear to provide a mechanism to control Itgb1 expression through differential regulation of stability and translation efficiency that can be finely tuned during mammary gland development.

\section{Acknowledgments}

We thank members of IFIBYNE - CONICET - FCEN - UBA for sharing equipment and technical advice. We want to thank Dr Bin Tian from the Department of Biochemistry and Molecular Biology, UMDNJ - New Jersey Medical School for providing the original $\mathrm{pRiG}$ reporter and for helpful advice regarding its use. We want to thank Instituto de Investigación en Biomedicina de Buenos Aires (IBioBA), CONICET, Partner Institute of the Max Planck Society, Argentina for sharing equipment. This work was supported by grants and fellowships awarded by: University of Buenos Aires, CONICET and ANPCyT, Argentina.

\section{Statement of author contribution}

Conceived and designed the experiments: JN, AG, ECK and OAC. Performed the experiments: JN, AG, VS, EW, MA. Analyzed the data: JN, AG, MA, ECK and OAC. Helped set up techniques and/or discuss preliminary ideas and data: MSD and JL. Contributed reagents/materials/analysis tools: MSD, EW, JL and MA. Wrote the paper: JN, ECK and OAC.

\section{References}

1. Schwartz, M.A. (1997) Integrins, oncogenes, and anchorage independence. J. Cell Biol. 139, 575-578.

2. Hynes, R.O. (2002) Integrins: bidirectional, allosteric signaling machines. Cell. 110, 673-687.

3. Brakebusch C, Fassler R. (2005) beta 1 integrin function in vivo: adhesion, migration and more. Cancer Metastasis Rev. 24(3), 403-411.

4. Taddei I, Faraldo MM, Teuliere J, Deugnier MA, Thiery JP, Glukhova MA. (2003) Integrins in mammary gland development and differentiation of mammary epithelium. Journal of Mammary Gland Biology and Neoplasia, 8(4), 383-394.

5. Anderson R, Fassler R, Georges-Labouesse E, Hynes RO, Bader BL, Kreidberg JA, Schaible K, Heasman J, Wylie C. (1999) Mouse primordial germ cells lacking eta1 
Alternative polyA of Itgb1 in mammary gland

integrins enter the germline but fail to migrate normally to the gonads. Development, 126(8), 1655-1664.

6. Fassler R, Meyer M. (1995) Consequences of lack of beta 1 integrin gene expression in mice. Genes Dev. 9(15), 1896-1908.

7. Stephens LE, Sutherland AE, Klimanskaya IV, Andrieux A, Meneses J, Pedersen RA, Damsky CH. (1995) Deletion of beta 1 integrins in mice results in inner cell mass failure and peri-implantation lethality. Genes Dev. 9(15), 1883-1895.

8. Klinowska TC, Soriano JV, Edwards GM, Oliver JM, Valentijn AJ, Montesano R, Streuli CH. (1999) Laminin and betal integrins are crucial for normal mammary gland development in the mouse. Dev Biol, 215(1), 13-32.

9. Li N, Zhang Y, Naylor MJ, Schatzmann F, Maurer F, Wintermantel T, Schuetz G, Mueller U, Streuli CH, Hynes NE. (2005) Beta1 integrins regulate mammary gland proliferation and maintain the integrity of mammary alveoli. EMBO J. 24(11), 19421953.

10. Naylor MJ, Li N, Cheung J, Lowe ET, Lambert E, Marlow R, Wang P, Schatzmann F, Wintermantel T, Schuetz G. (2005) Ablation of betal integrin in mammary epithelium reveals a key role for integrin in glandular morphogenesis and differentiation. J Cell Biol, 171(4), 717-728.

11. Edwalds-Gilbert, G., Veraldi, K. L., and Milcarek, C. (1997) Alternative poly(A) site selection in complex transcription units: means to an end? Nucleic Acids Res. 25, 2547-2561.

12. Chabot, B. (1996) Directing alternative splicing: cast and scenarios, Trends Genet. 12, 472- 478 .

13. Garneau NL, Wilusz J, Wilusz CJ. (2007) The highways and byways of mRNA decay. Nat Rev Mol Cell Biol. 2, 113-26.

14. Hynes NE, Taverna D, Harwerth IM, Ciardiello F, Salomon DS, Yamamoto T, Groner B. (1990) Epidermal growth factor receptor, but not c-erbB-2, activation prevents lactogenic hormone induction of the beta-casein gene in mouse mammary epithelial cells. Mol Cell Biol. 10(8), 4027-4034.

15. Quaglino A, Salierno M, Pellegrotti J, Rubinstein N, Kordon EC. (2009) Mechanical strain induces involution-associated events in mammary epithelial cells. BMC Cell Biol. 10, 55.

16. Y You, C Y Chen and A B Shyu. (1992) U-rich sequence-binding proteins (URBPs) interacting with a 20-nucleotide U-rich sequence in the $3^{\prime}$ untranslated region of c-fos mRNA may be involved in the first step of c-fos mRNA degradation. Mol. Cell. Biol. 12(7), 2931.

17. Zhe Ji, Wenting Luo, Wencheng Li, Mainul Hoque, Zhenhua Pan, Yun Zhao and Bin Tian. (2011) Transcriptional activity regulates alternative cleavage and polyadenylation. Molecular Systems Biology 7, 534.

18. Saeed AI, Sharov V, White J, Li J, Liang W, Bhagabati N, Braisted J, Klapa M, Currier T, Thiagarajan M, Sturn A, Snuffin M, Rezantsev A, Popov D, Ryltsov A, Kostukovich E, Borisovsky I, Liu Z, Vinsavich A, Trush V, Quackenbush J. (2003) TM4: a free, open-source system for microarray data management and analysis. Biotechniques. 34, 374-8.

19. Gattelli A, Zimberlin MN, Meiss RP, Castilla LH, Kordon EC. (2006) Selection of early-occurring mutations dictates hormone-independent progression in mouse mammary tumor lines. J Virol. 80(22), 11409-15. 
Alternative polyA of Itgb1 in mammary gland

20. Solange Soulier, Jean-Luc Vilotte, Phillip J. L'Huillier and Jean-Claude Mercier. (1996) Developmental regulation of murine integrin pl subunit- and Hsc73-encoding genes in mammary gland: sequence of a new mouse Hsc73 cDNA. Gene. 172, 285289.

21. Brennan CM, Steitz JA. (2001) HuR and mRNA stability. Cell Mol Life Sci. 58(2), 266-77.

22. Zhe Ji, Ju Youn Lee, Zhenhua Pan, Bingjun Jiang, and Bin Tian. (2009) Progressive lengthening of 3 'untranslated regions of mRNAs by alternative polyadenylation during mouse embryonic development. PNAS. 106(17), 7028-7033. 23. Pontier SM, Muller WJ. (2009) Integrins in mammary-stem-cell biology and breastcancer progression--a role in cancer stem cells? J Cell Sci. 122, 207-214.

24. Taddei I, Deugnier MA, Faraldo MM, Petit V, Bouvard D, Medina D, Fassler R, Thiery JP, Glukhova MA. (2008) Beta1 integrin deletion from the basal compartment of the mammary epithelium affects stem cells. Nat Cell Biol. 10(6), 716-722.

25. McSherry EA, McGee SF, Jirstrom K, Doyle EM, Brennan DJ, Landberg G, Dervan PA, Hopkins AM, Gallagher WM. (2009) JAM-A expression positively correlates with poor prognosis in breast cancer patients. Int J Cancer. 125(6), 1343-51. 26. Ruea-Yea Huang · Margot M. Ip. (2001) Differential expression of integrin mRNAs and proteins during normal rat mammary gland development and in carcinogenesis. Cell Tissue Res. 303, 69-80.

27. Neelanjan Mukherjee, Patrick J Lager, Matthew B Friedersdorf, Marshall A Thompson and Jack D Keene. (2009) Coordinated posttranscriptional mRNA population dynamics during T-cell activation. Molecular systems biology. 5, 288.

28. Yan W, Zhang Y, Zhang J, Cho S-J, Chen X. (2012) HuR Is Necessary for Mammary Epithelial Cell Proliferation and Polarity at Least in Part via DNp63. PLoS ONE. 7(9), 45336.

29. Kriegel AJ, Liu Y, Fang Y, Ding X, Liang M. (2012) The miR-29 family: genomics, cell biology, and relevance to renal and cardiovascular injury. Physiol Genomics. 44(4), 237-44.

30. Li G, Luna C, Qiu J, Epstein DL, Gonzalez P. (2010) Targeting of integrin beta1 and kinesin 2alpha by microRNA 183. J Biol Chem. 285(8), 5461-71.

31. Li KK, Pang JC, Ching AK, Wong CK, Kong X, Wang Y, Zhou L, Chen Z. Ng HK. (2009) miR-124 is frequently down-regulated in medulloblastoma and is a negative regulator of SLC16A1. Hum Pathol. 40(9), 1234-43.

32. Avril-Sassen S, Goldstein LD, Stingl J, Blenkiron C, Le Quesne J, Spiteri I, Karagavriilidou K, Watson CJ, Tavaré S, Miska EA, Caldas C. (2009) Characterisation of microRNA expression in post-natal mouse mammary gland development. BMC Genomics. 20, 10:548.

33. Young LE, Moore AE, Sokol L, Meisner-Kober N, Dixon DA. (2012) The mRNA stability factor HuR inhibits microRNA-16 targeting of COX-2. Mol Cancer Res. 10(1), 167-80.

34. Bin Tian, Jun Hu, Haibo Zhang1 and Carol S. Lutz. (2005) A large-scale analysis of mRNA polyadenylation of human and mouse genes. Nucleic Acids Research. 33, 201-212.

35. Kornblihtt AR. (2007) Coupling transcription and alternative splicing. Adv Exp Med Biol. 623, 175-89. 
Alternative polyA of Itgb1 in mammary gland

36. Zhe Ji, Ju Youn Lee, Zhenhua Pan, Bingjun Jiang, and Bin Tian. (2009) Progressive lengthening of 3'untranslated regions of mRNAs by alternative polyadenylation during mouse embryonic development. PNAS. 106(17), 7028-7033. 37. Alonso CR. (2012) A complex 'mRNA degradation code' controls gene expression during animal development. Trends Genet. 28(2), 78-88.

\section{Figure legends}

Figure 1. Genomic structures and expression of the mouse integrin $\beta 1$ gene analized using EST and SAGE databases. (A) Schematic representation of the mouse Itgb1 3'UTR region. Two hundred and eighty three mouse Itgb1 3'-reads EST ends (Unigene Mm.263396) were mapped on the 3'UTR region, its analysis identified two transcript variants (cluster 1 and cluster 2) corresponding to two polyadenylation sites. (B) Tissue-specific expression of both Itgb1 variants (Itgb1-S and Itgb1-L) among 10 different mouse tissues according to the EST data. (C) Hierarchical clustering analysis of Itgb1 variants expressed in different mouse tissues as indicated below based on the analysis of $269 \mathrm{mSAGE}$ libraries available at SAGEgenie database. The grayscale at the bottom of the picture is used to represent relative expression level based on numbers of transcripts (tags) per libraries. The values range from 0 (white) to 180 (black). The position of tissue specific libraries in the cluster is indicated as R: Retina, B: Brain, MG: Mammary Gland, U: Urogenital tract, VE: Vascular Endothelium.

Figure 2. Identification of the 3'UTR-differential Itgbl mRNA isoforms. (A) Schematic representation of the Itgb1 gene of BALB/c mice (NCBI: GenBank accession no. NM_010578.2). The start codon is located in exon 1 and the stop codon is in exon 17. Grey boxes are 5' and 3'UTR regions, the latter including polyA sequences (PAS1 and PAS2) and cleavage and polyadenylation sites (pA1 and pA2, arrows). Black boxes denote exons and lines, introns. The graph is not shown to scale. (B) Schematic representation of the cloning and sequencing protocol. (C) Analysis of 3'RACE PCR products by electrophoresis in an EtBr-stained agarose gel identifies two species of Itgb1 messenger RNAs with 3'UTRs of different length (long, 1400bp and short, 750bp) indicated by arrows. RNA was obtained from BALB/c female mice normal mammary glands (NMG), either virgins in estrum (V) or lactating (L) and 4 mammary tumors from independent in vivo tumor lines $(1,2,3$ and 4$)$ induced by mouse mammary tumor virus MMTV. (D) Sequence of Itgb1 3'UTR showing the two PAS sequences (bold underlined), the 2 ARE sequences (grey boxes and bold) and primers (arrows) used for Real time PCR. Exonic sequences shown in "italic bold" font. (E) Diagram showing the two Itgb1 isoforms with differences at the 3'UTR, polyadenylation signals (PAS1 and PAS2, dark grey bars), sites of cleavage and polyadenylation (pA1 and pA2, vertical arrows), ARE regions (white bars) and location of the primers for the qPCRs (horizontal arrows).

Figure 3. Cleavage and Polyadenylation activity directed by the Itgb1 polyA site 1 (pA1). (A) Diagram showing relevant features of the $\mathrm{pRiG}$ vector. $\mathrm{pCMV}$, human cytomegalovirus (CMV) immediate early promoter; RFP, coding region for red fluorescent protein; MCS, multiple cloning sites; IRES, internal ribosome entry site; 
Alternative polyA of Itgb1 in mammary gland

EGFP, coding region for enhanced green fluorescent protein; 77S.AE, 77S.AE polyadenylation site and Itgb1.pA1, Itgb1 polyadenylation site 1 in its wild type and mutated versions, the core sequences of the cloned fragments are shown in detail. (B) FACS analysis of $\mathrm{HC} 11$ cells transfected with different pRiG vectors, as indicated above each graph. pRiG: pRiG empty vector; $p$ RiG-77S.AE: pRiG vector with an 77S.AE polyA site sequence inserted in the MCS; pRiG-Itgb1.pA1: pRiG vector with a sequence containing the region surrounding PAS1 of mouse Itgb1 inserted in the MCS, pRiG-Itgb1.pA1mut: pRiG vector with a mutated sequence containing the region surrounding PAS1 of mouse Itgb1 inserted in the MCS. The intensity of red fluorescence (y-axis) and the intensity of green fluorescence (x-axis) were read as indicated in Materials and Methods. Each dot represents a cell. One representative experiment out of four performed is shown. Cumulative fraction curves of ratio of green fluorescence to red fluorescence for cells are shown (lower graph). Only cells with red fluorescence intensity higher than background were selected for analysis. (C) Quantitative measurement of the ratio of red to green fluorescence as read in the upper-left and lower-right quadrants respectively, calculated as the average of four different experiments. $\# \mathrm{p}<0,05$ One-way ANOVA, followed by a Tukey post-test.

Figure 4. Relative expression of different Itgb1 mRNA species during normal mammary gland development. (A) Itgb1 and Actin protein levels from mammary glands (NMG) of virgin, pregnant, lactating and post-involution (INV 72h) female mice were analyzed by Western blot and quantified by the image $\mathrm{J}$ programme calculated as the average of three different experiments. \# $p<0,05$ One-way ANOVA, followed by a Tukey post-test. (B) Total Itgb1 mRNAs levels were determined in the same RNA samples by quantitative RT-PCR (qPCR). \# $p<0,05$ One-way ANOVA, followed by a Tukey post-test. (C) The expression of the isoforms of the mRNA of Itgb1, Itgb1-L (white columns) and Itgb1-S (black columns) mRNAs levels were determined in the same RNA samples by qPCR. (D) qPCR assays for Itgb1 mRNA isoforms short and long (Itgb1-S, black columns and Itgb1-L, white columns) present in progressive post-involution mammary glands (INV 24h, 48h and $72 \mathrm{~h}$ ). ${ }^{*} \mathrm{p}<0,05$ Student t-test. (E) Same as in (D) using HC11 cells subject to mechanical stretching as a source of RNA. Gapdh mRNA was used as normalization control in all the qPCRs. ${ }^{*} \mathrm{p}<0,05$ Student t-test.

Figure 5. Biological relevance of the Itgb1-S mRNA isoform. (A) Western blot analysis of protein extracts from HC11 cell lines transfected with diferent ShRNAs plasmids and selected by antibody resistance: ShRNA control or scramble (Shctrl), ShRNA against Itgb1-T mRNA (ShItgb1-T), and ShRNA against Itgb1-L mRNA (ShItgb1-L). Itgb1, Cyclin D1 and Actin antibodies were evaluated. (B) qPCR assays to measure the levels of Itgb1 mRNA isoforms in cell lines that express the different shRNAs indicated. \# $\mathrm{p}<0,05$ One-way ANOVA, followed by a Tukey post-test. (C) $3 \mathrm{D}$ cultures of $\mathrm{HC} 11$ cell lines were achieved by seeding cells in Matrigel coated plates. Representative optic microscope images of are shown

Figure 6. Endogenous expression of Itgb1 isoforms during differentiation of HC11 cells. (A) and (B) Representative experiments of mRNA stability assays performed three times independently. HC11 cells were cultured under proliferative and differentiation conditions. Actinomycin $\mathrm{D}(5 \mathrm{ug} / \mathrm{ul})$ was added $(\mathrm{t}=0)$, and mRNA levels of Itgb1 mRNA isoforms were analized by qPCR following a time course 
Alternative polyA of Itgb1 in mammary gland

(hours). Samples corresponding to cells proliferating or differentiated are depicted with dashed lines and continuous lines respectively. Primers directed to detect the Itgb1-L isoform (A) or the Itgb1-S isoform (B) were used. Gapdh mRNA was used as normalization control. (C) Expression of HuR mRNA in HC11 cells under proliferative and differentiation conditions as shown by qPCR analysis. Gapdh mRNA was used as normalization control. ${ }^{*} \mathrm{p}<0,05$ Student t-test. (D) HuR protein expression levels in the same samples. (E) Levels of HuR mRNA expression were measured by qPCR using RNA from normal mammary glands (NMG) of female mice virgins in estrum $(\mathrm{V})$, pregnant $(\mathrm{P})$, lactating (L) or post-involution (INV 72h). * $\mathrm{p}<0,05$ Student t-test. (F) HuR and Actin protein levels were analyzed by Western blot in the same samples.

Figure 7. (A) Diagram showing part of the last exon (\# 17) corresponding to the 3'UTR of the Itgb1 gene. Different segments were cloned downstream of a luciferase reporter gene (black box) driven by a constitutive promoter or under the control of a Tetracycline responsive promoter (Tet-off) (not shown). Two reporter constructs were generated: "3'UTR Short" includes a segment corresponding to the short form of the Itgb1 mRNA and "3' UTR D" includes the differential segment. Numbers 29, 124 and 183 indicate binding sequences for miRNAs. White bars indicate ARE sequences and arrows sites of cleavage and polyadenylation that produce both mRNA forms: Itgb1-L and Itgb1-S preceded by 2 alternative polyadenylation signals (PAS1 y PAS2) indicated by dark grey bars. (B) mRNA stability assay from cells transfected with a reporter plasmid containing the " 3 ' UTR D" cloned downstream of the Luciferase open reading frame under the control of a Tetracycline responsive promoter (Tet-off). Cells were co-transfected with a HuR expression vector (solid line) or with empty plasmid (dashed line).\# $\mathrm{p}<0,05$ One-way ANOVA, followed by a Tukey post-test. (C) A radiolabeled probe containing the Itgb1 ARE1 sequence (100-nt probe) was incubated with 5-7 ug of HC11 protein lysate and mobility shift assays were carried out. Antibodies, recombinant proteins and unlabeled probes were added to the mixture before the addition of the labelled probe as indicated. (D) The Itgb1 ARE1 100-nt probe was incubated with 5-7 ug of protein lysate from normal mammary glands at different developmental stages as indicated: BALB/c female mice virgins in estrum (V), pregnant (P), lactating (L) or 72 hs post-involution (I72). Bands "a" to "c" denote representative shifted complexes and horizontal arrows indicate the HuR antibody super-shifted position. (E) HC11 cells were transfected with SiRNA control (Si control) or SiRNA HuR (Si HuR). Western blots (inset) and qPCR were performed in order to measure the levels of expression of HuR protein and mRNA respectively. * $\mathrm{p}<0,05$ Student t-test. (F) HC11 cells were transfected with the same SiRNAs and cultured under proliferative conditions. Actinomycin D $(5 \mathrm{ug} / \mathrm{ul})$ was added $(\mathrm{t}=0)$ and mRNA levels of Itgb1-L mRNA isoform was analized by qPCR following a time course (3hours). Samples corresponding to cells tranfected with Si control or Si HuR are depicted with dashed lines and continuous lines respectively. ${ }^{*} \mathrm{p}<0,05$ Student $\mathrm{t}-$ test. (G) Luciferase activity measured in HC11 cells transfected by the indicated reporter constructs driven by a constitutive promoter as measured by a luminometer.* $\mathrm{p}<0,01$ Student t-test. Luciferase activity values were normalized to the corresponding mRNA levels to obtain translation efficiencies. 
A)

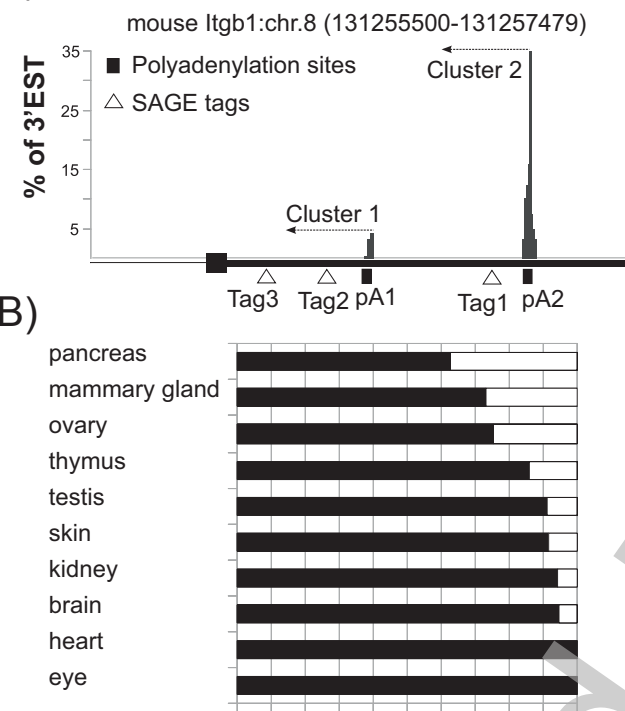

0102030405060708090100 Itgb1-L Expression (\%) $\square$ Itgb1-S
Figure 1

C)

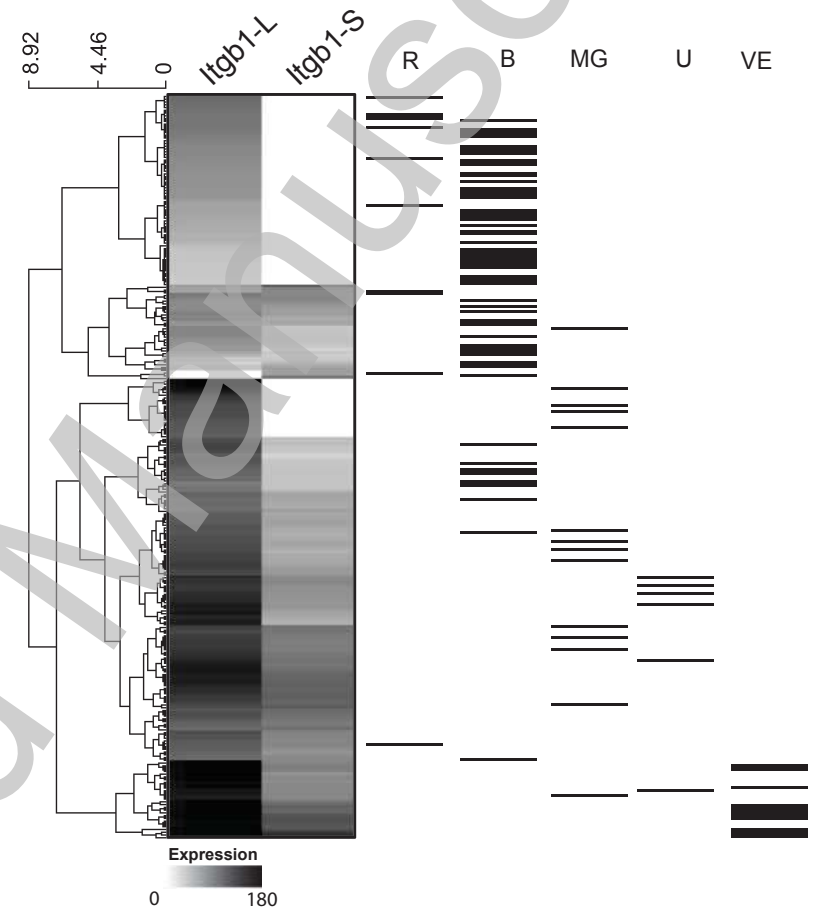


A)

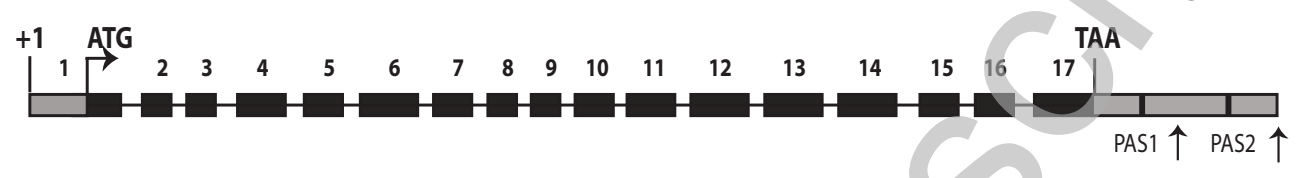

B)

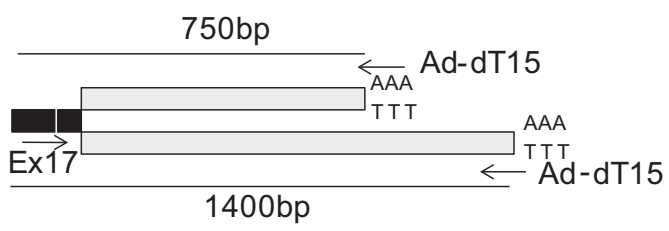

D)

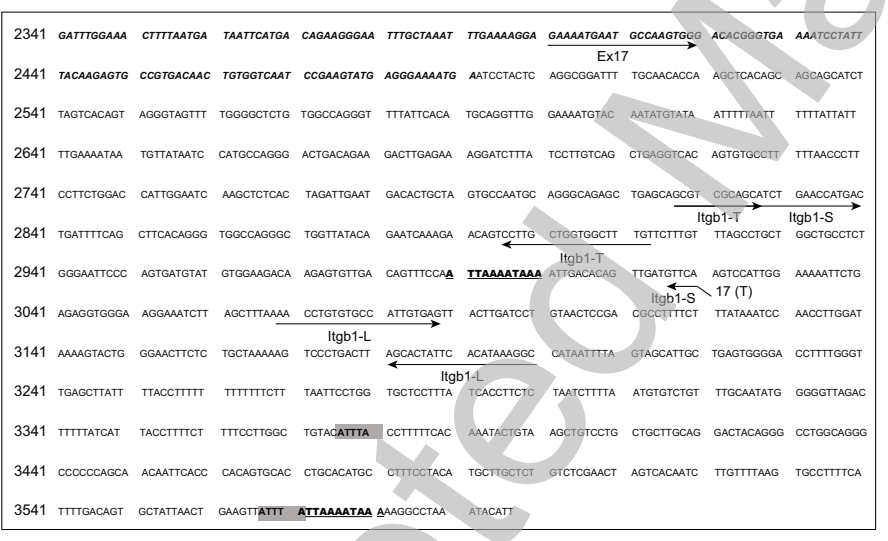

C)

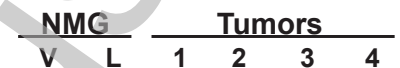
$1400 \mathrm{bp}$ 750 bp
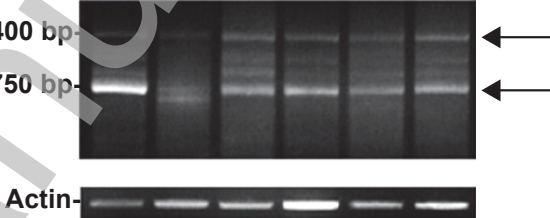

E)

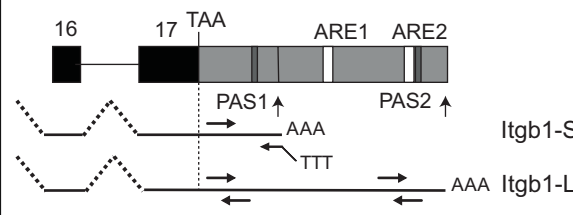


A)
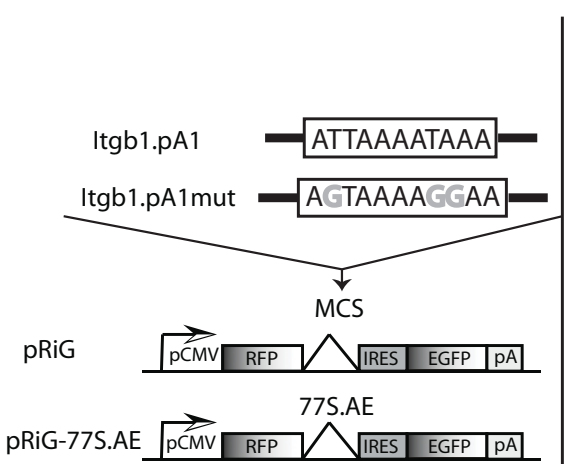

C)

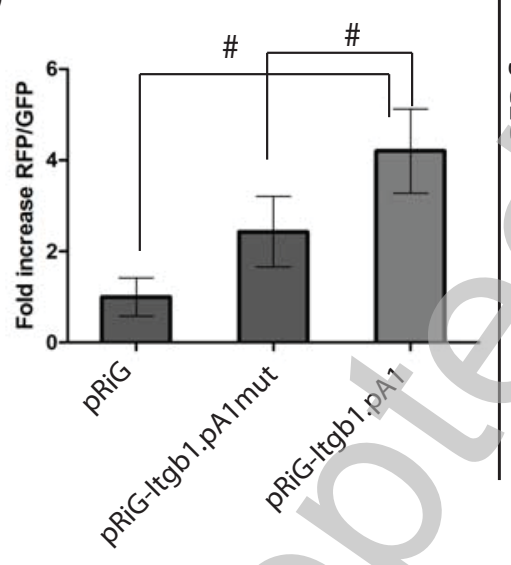

B)

Figure 3
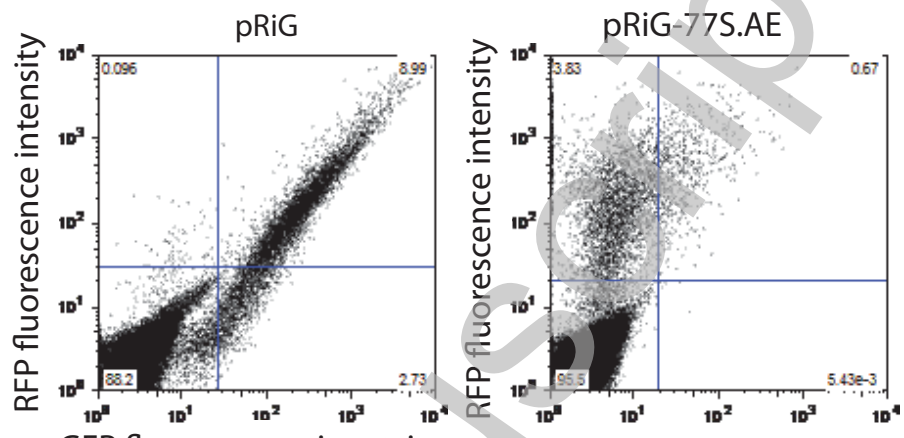

GFP fluorescence intensity pRiG-Itgb1.pA1mut
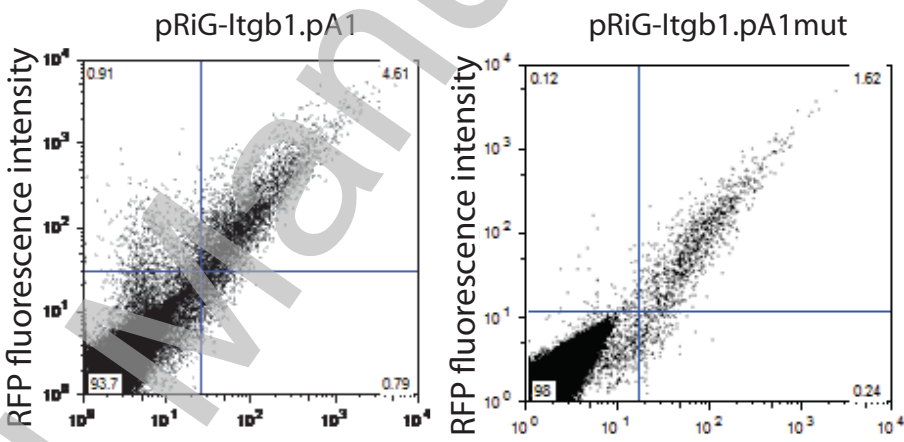

GFP fluorescence intensity

GFP fluorescence intensity EGFP/RFP

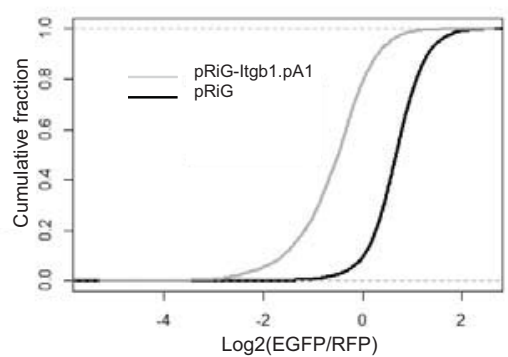


A)
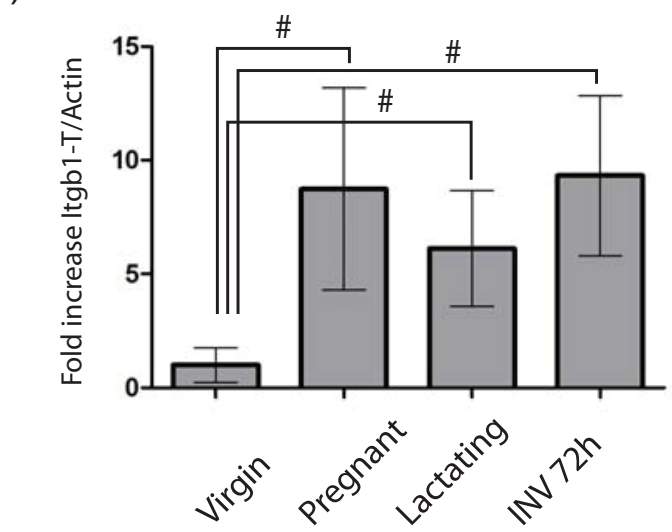

Itgb1

Actin

C)

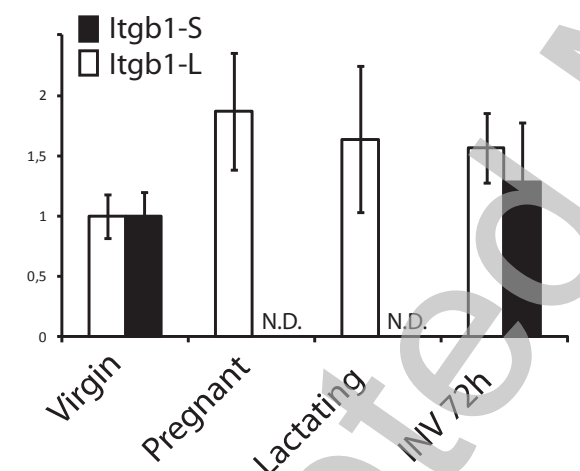

B)

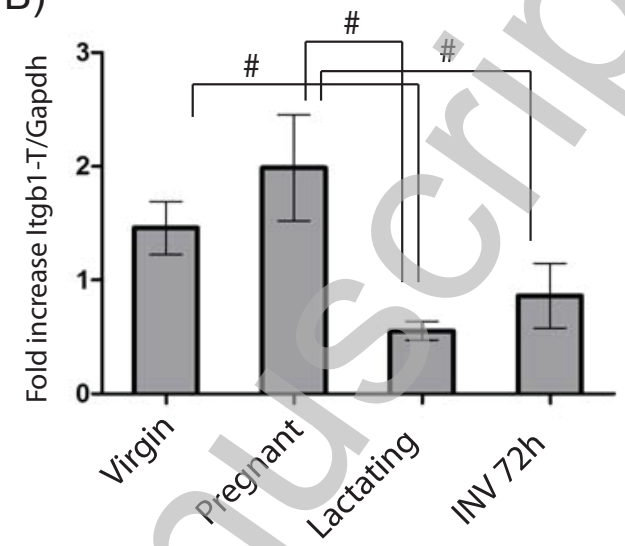

D)

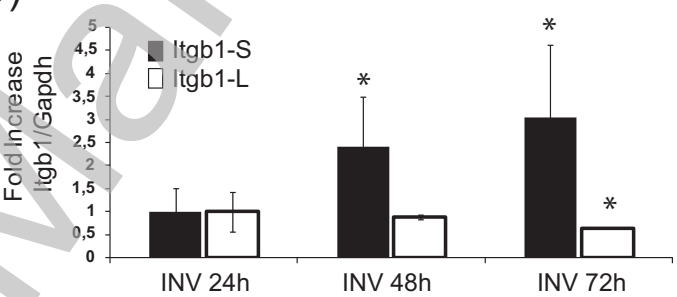

E)

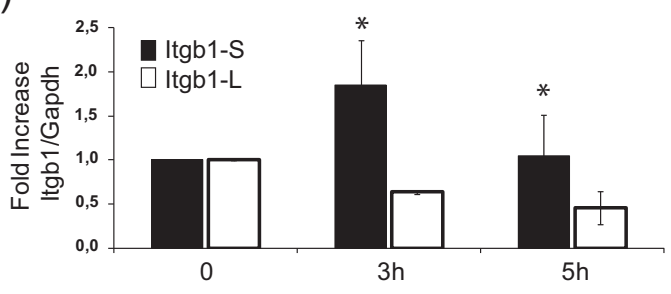


A)

Figure 5

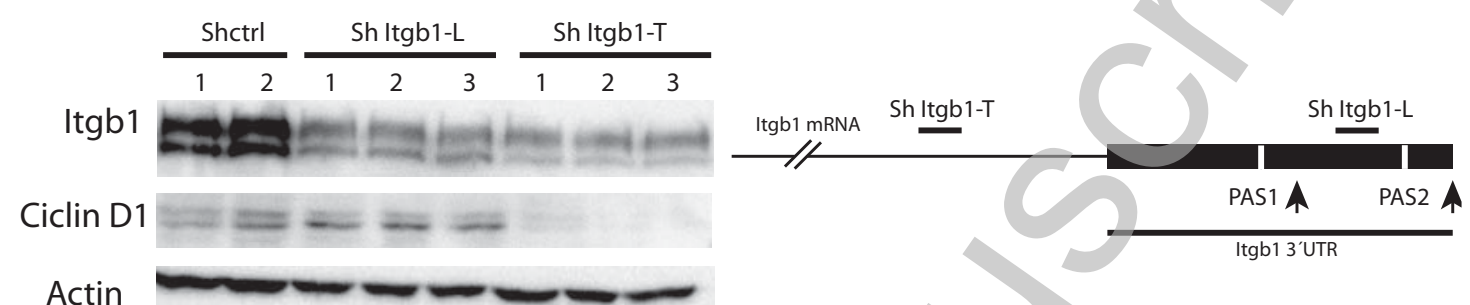

B)
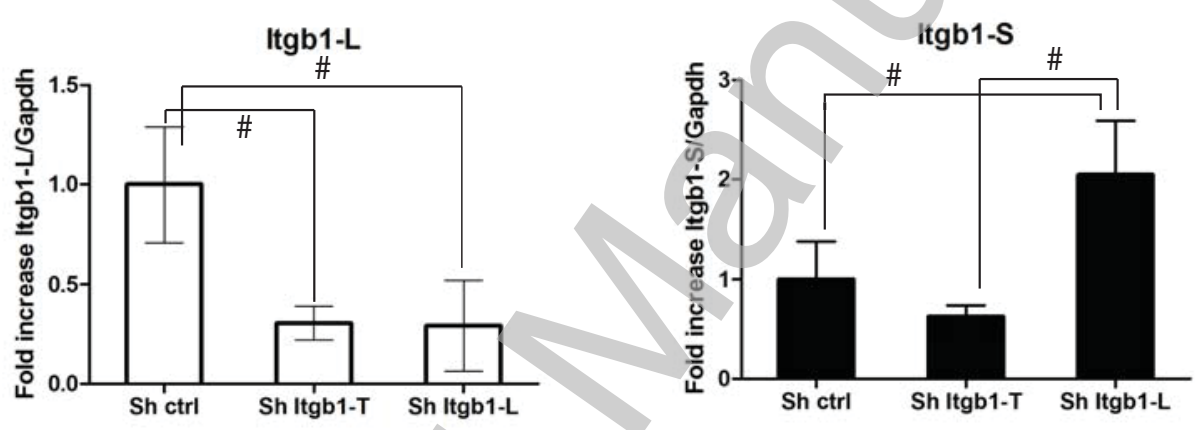

C)

Sh ctrl

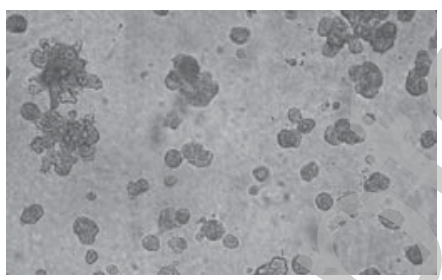

Sh Itgb1-T

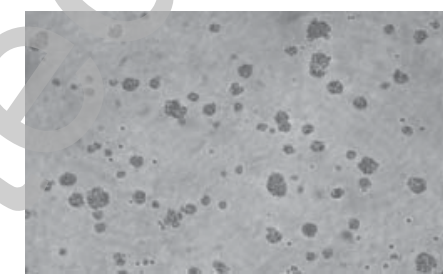

Sh Itgb1-L

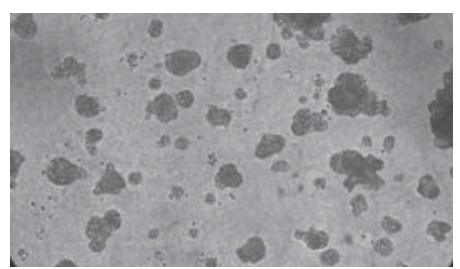


A)

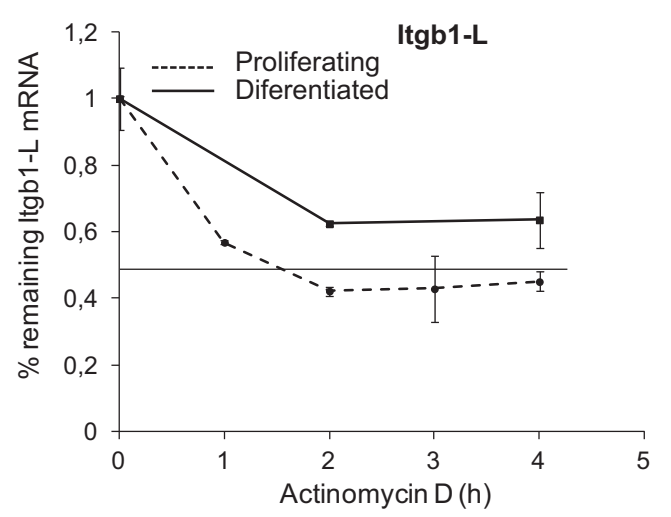

C)

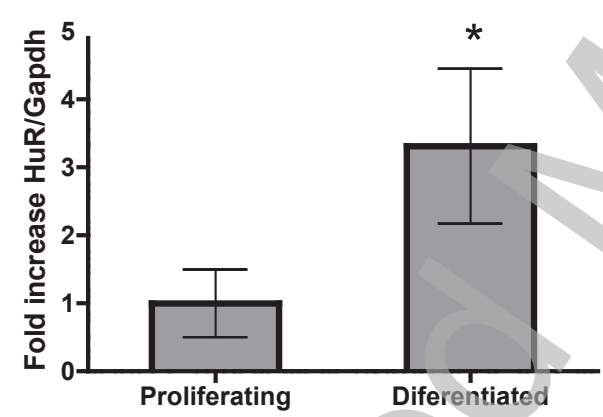

E)

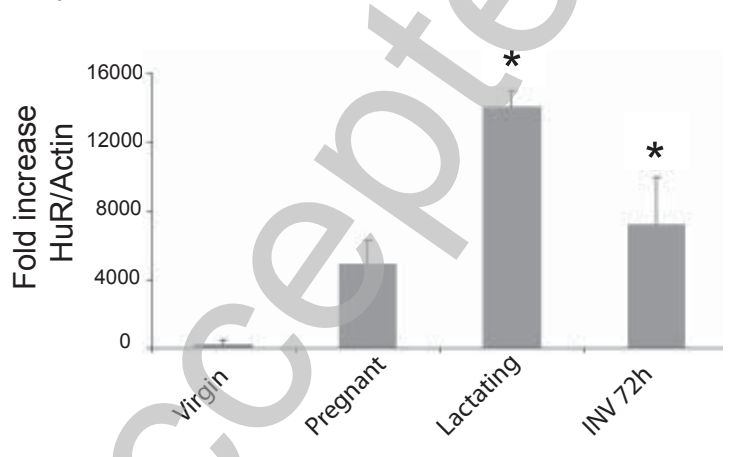

B)

Figure 6

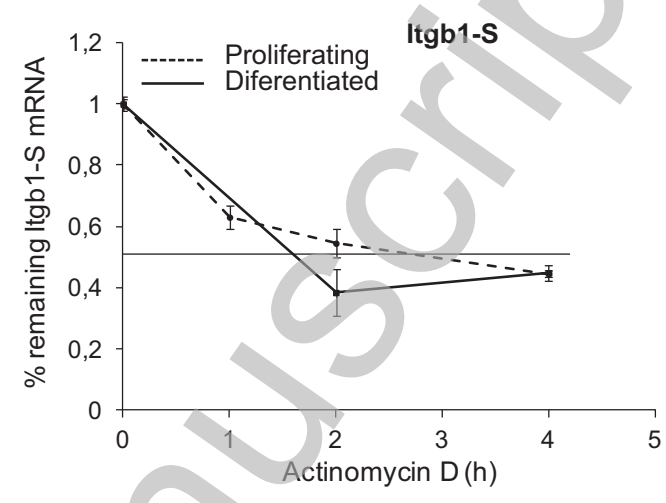

D)

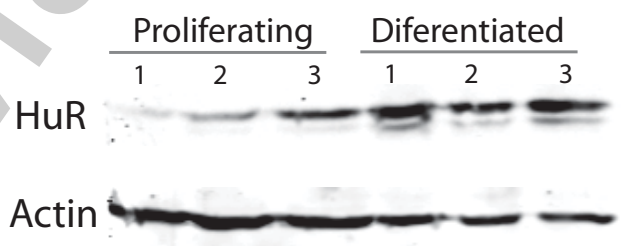

F)

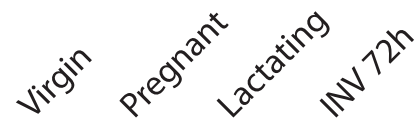

HuR

Actin 
A)

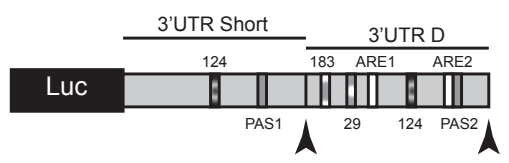

C)

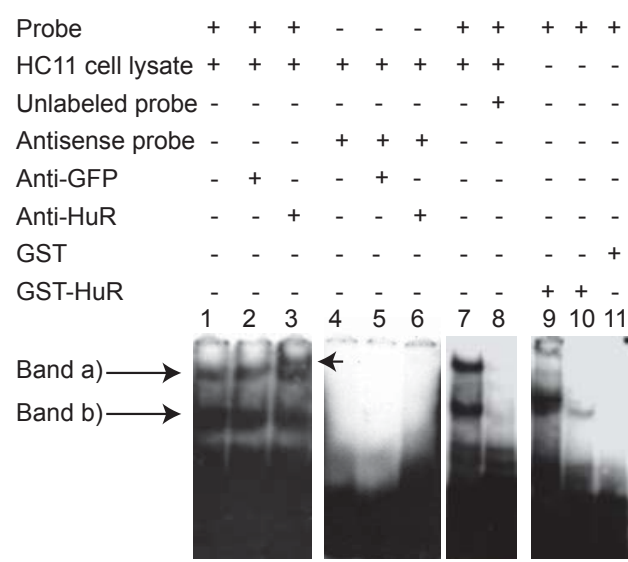

E)

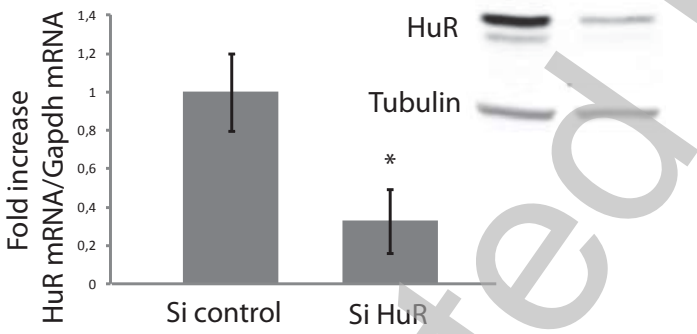

F)

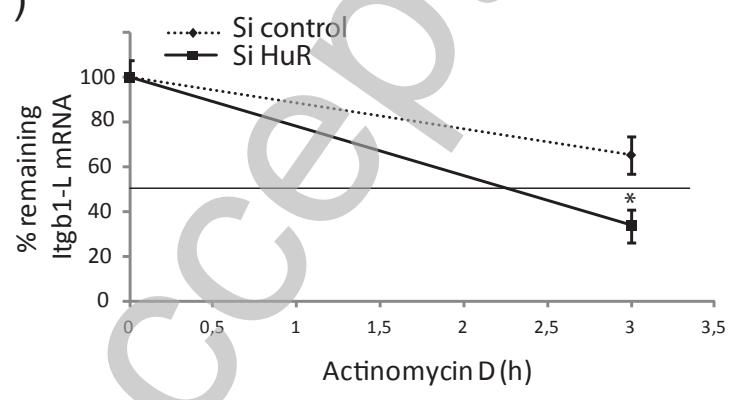

B)

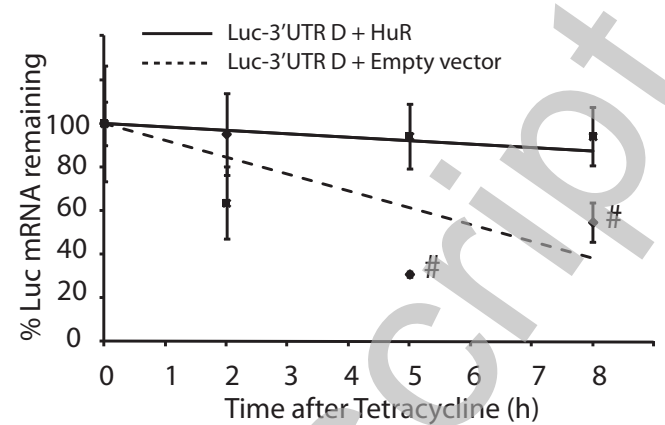

D)

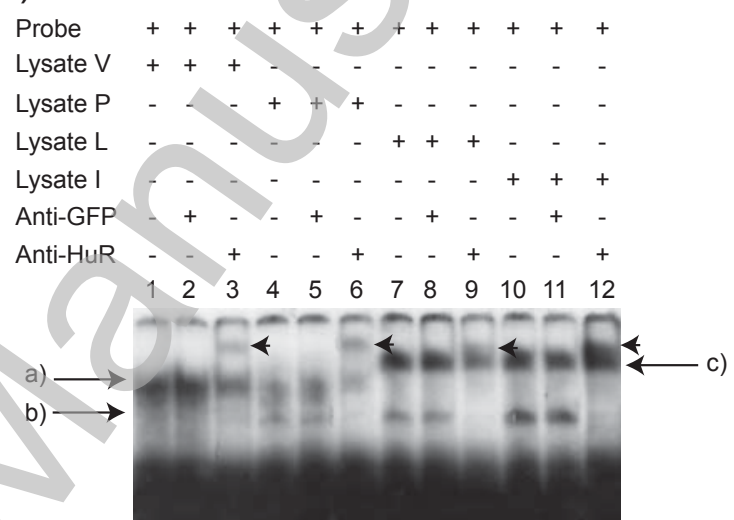

G)
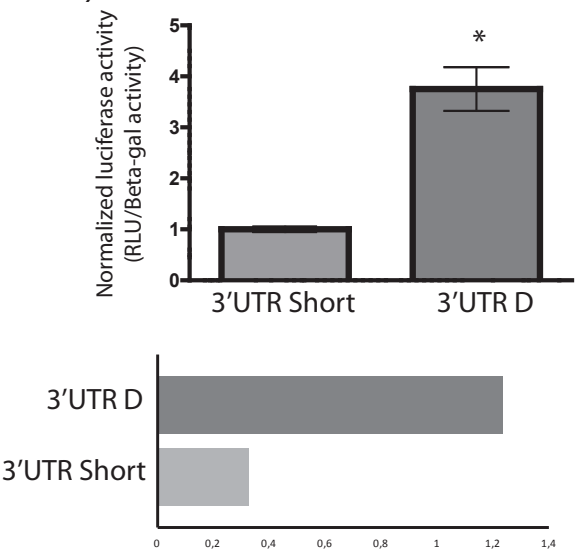

Translation efficiency (Luciferase activity (RLU/Beta-gal activity)) /(Luc mRNA levels (Luc mRNA/Beta-gal mRNA)) 